\title{
Study on the Control Performance of Rainwater Quality in the Ini- tial Stage of Urban Runoff by New Environmentally Friendly Rainwater Pass
}

\author{
Xinli Cai ${ }^{1}$, Qiaodan Zhang ${ }^{1}$, Bai Sun ${ }^{1,2}$, ZhenShan Liu ${ }^{3}$, ShuGuang Zhu ${ }^{1,2 *}$ \\ ${ }^{1}$ College of Environmental and Energy Engineering, Anhui Jianzhu University, Hefei 230601, China. \\ ${ }^{2}$ Key Laboratory of Huizhou Architecture in Anhui province, Anhui Jianzhu University, Hefei, 230601, China. \\ ${ }^{3}$ Anhui Yajing Rainwater Utilization Technology company, Hefei, 230601, China.
}

\begin{abstract}
In view of the current situation of gully in China, new type of environmentally-friendly gully with anti-mosquito and odor-reducing function has been developed. The control performance of new gully on the initial rainwater pollutants under the condition of different filter material ratio and filter bag thickness. The results show that the new environmentally-friendly gully has better removal effect of various pollutants in the initial rainwater under the condition of $1: 3$ filter material and $3 \mathrm{~cm}$ filter bag. The removal rate of SS is over $80 \%$, total nitrogen the removal rate is above $75 \%$, and the removal rate of COD and total phosphorus is over $70 \%$.
\end{abstract}

\section{Introduction}

After years of continuous efforts, China's urban drainage pipe network structure has basically taken shape, but the construction plan is not systematic. The main drain pipe and main pipe are relatively complete, the branch pipe and the collecting pipe network are incomplete, and the construction quality is relatively rough, which seriously affects the sewage intercepting pipe and also causes the rainwater pipeline to fail to play its due role $[1,2]$.

In the initial prevention and control of rainwater, the gully is the most front-end structure for storing rainwater, and effective measures can improve rainwater pollution [3]. The gully is a facility for collecting surface water from the pipeline drainage system. The traditional gully cannot intercept the pollutants in the water, and with the changes of the urban ecological environment, some mosquitoes have also changed their living environment $[4,5]$. It is easy to cause odors in the rain inlet, flying insects such as flies and mosquitoes, reptiles coming out from the gully, and some places are constantly suffering from rodents, which seriously affect the urban living environment [6]-[10]. It is easy to cause odors in the rain inlet, flying insects such as flies and mosquitoes, reptiles coming out from the gully, and some places are constantly suffering from rodents, which seriously affect the living environment of the city [11].

In order to solve the above problems, a new environmentally friendly gully with anti-mosquito and odor-reducing functions has been developed. This paper will study the new gully structure and the performance of the rainwater outlet to control the pollutants in the initial rainwater.

\section{Material and methods}

\subsection{Experimental apparatus}

The design of the new environmentally-friendly gully is as follows: This test device is a new type of environmentally friendly gully. The new gully is equipped with a sewage intercepting basket under the grid, which not only intercepts large substances but also filters rainwater. Compared with the traditional gully, the water louver is added under the intercepting basket, and the filter bag is installed under the louver of the water. Under the adsorption of the filter bag containing the filter material, the gully has the characteristics of controlling the characteristics of the runoff pollutant. A protective cover is installed at the bottom of the gully. When the rainfall is large, the sediment is prevented from being stirred up and the filter material is blocked.

\subsection{Experimental methods and equipment}

The indicator test method adopts the national standard method or the environmental protection industry standard. The rainwater quality test indicators include SS (Suspended solid), CODcr (chemical oxygen demand), TN (Total Nitrogen), and TP (Total Phosphorus) indicators.

The main equipment includes ultrapure water machine, analytical balance, COD digestion instrument, COD analyzer, UV spectrophotoeter.

\footnotetext{
* Corresponding author E-mail address: bsun@mail.ustc.edu.cn 286537056@qq.com (S. G. Zhu)
} 


\subsection{Rainwater structure design}

The design of the gully is based on the traditional gully, especially for the interception of pollutants such as leaves and large particles, to prevent mosquito breeding and odor prevention, and to design a relatively new gully structure. The gully consists of a shell, a rainwater bi, a sewage intercepting basket, a water louver, a filter bag and a muddy area.

According to the scene used by the gully, we designed two sizes of gullies, the inner diameter of which is $680 \times$ $380 \times 960 \mathrm{~mm}$ and $530 \times 380 \times 960 \mathrm{~mm}$. There are two types of rainwater rafts: A-type gully $(750 \times 450 \mathrm{~mm})$ and B-type gully $(600 \times 400 \mathrm{~mm})$, and the opening ratio is $34 \%$. Type A gully rainwater rafts are mainly used for urban main roads. Type B gully rainwater rafts are mainly used for urban secondary trunk roads and residential interior roads and square green spaces. The internal structure of the A-type gully is consistent with the structure of the Btype gully. There is only a difference in size. The structure of the A-type gully is described below in detail.

Type A gully rainwater rafts are mainly used for urban main roads. Type B gully rainwater rafts are mainly used for urban secondary trunk roads and residential interior roads and square green spaces. The internal structure of the A-type gully is consistent with the structure of the Btype gully. There is only a difference in size. The structure of the A-type gully is described below in detail.

Type A gully is mainly used for newly built roads, green spaces, etc. The size of the gully is designed according to the existing "rain gully" (16S518) size and the size is $450 \mathrm{~mm} \times 750 \mathrm{~mm}$.

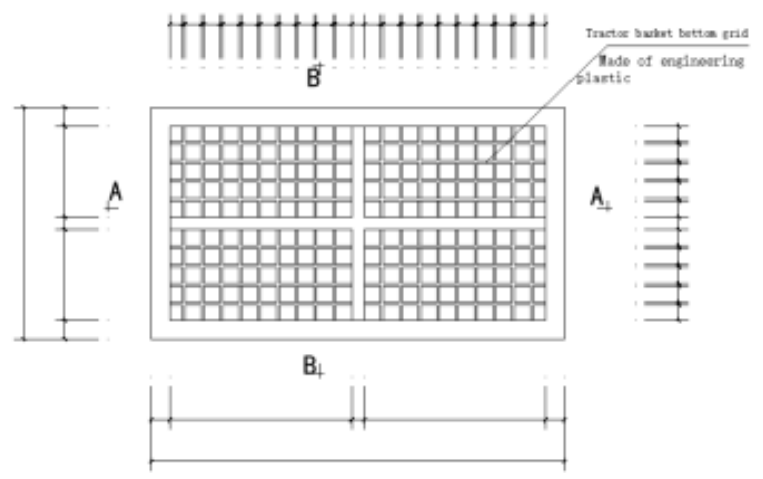

Fig.1. Plan of the interception basket

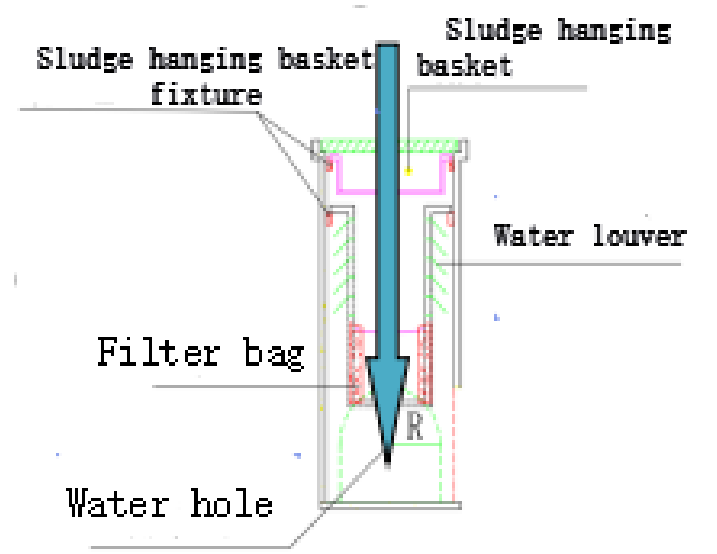

Fig. 2. (a). Small rainfall working chart

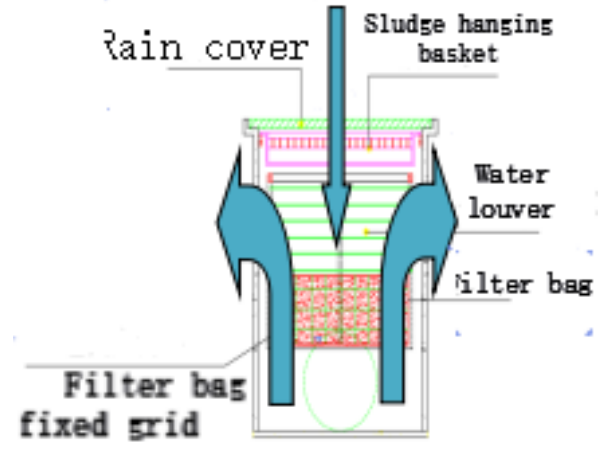

Fig. 2(b). heavy rainfall working chart

A filter bag is placed in the longitudinal direction at the bottom of the sinking area. In the sinking mud area, a set of water-passing louvers are arranged in the longitudinal direction from the bottom, and the water-passing louver material is made of engineering plastics, which can ensure flexible opening of the water-passing louvers during heavy rain. The water-passing louvers are mainly used to ensure the amount of heavy rain required by all the design during heavy rain, and can usually play a role in preventing mosquitoes and deodorants. The working principle is that when there is no rain, the water louvers use their own gravity to close the water louvers to achieve the anti-mosquito and odor-reducing function; in the initial rain, the rainwater directly falls into the sinking mud area because the rainwater is small. The content of ammonia nitrogen pollutants in rainwater is very high. The initial rainwater is filtered through the filter bag at the bottom of the muddy soil area to remove the pollutants such as ammonia nitrogen in the initial rainwater. The sludge in the sinking mud area should be cleaned regularly, and the filter bag should be cleaned regularly to ensure the over-watering and the interception of pollutants such as SS.

\subsection{Development of rainwater filter material}

For the filter bag placed in the gully, considering the market conditions, economic benefits and problems encountered in subsequent use, it is preliminarily determined that the filter bag is brick type. The brick filter bag is prepared from three aspects of material selection, mesh number and ratio. The brick filter bag is prepared as follows: the raw materials (natural porous medium and activated carbon are mixed according to a certain ratio) are sieved by a sieving machine to remove materials and impurities whose particle diameters do not meet the requirements, and after the sieving is finished, the methods such as washing and ultrasonic are used. Remove impurities from the surface of the material. The cleaned and ultrasonicated materials were placed in an oven at a constant temperature of $50{ }^{\circ} \mathrm{C}$ for $10 \mathrm{~h}$. After the end of drying, it was naturally cooled to room temperature. The raw materials and the binder are mixed in a certain ratio, and the stirring is continued vigorously until the binder is uniformly mixed with the raw materials. Pour the mixture into the mold, spread the mold, and shake evenly. It is pressed with a physical object and placed at room temperature for 12 hours. The brick filter material has a thickness of $3 \mathrm{~cm}$, and the length and width 
of the prepared brick type filter material are vertically placed on the side of the container.

The brick type permeable filter material is permeable by natural porous medium and activated carbon processing. The two pieces of brick type permeable water permeable filter material are on both sides of the bottom, and it is desirable to realize the interception of some pollutants. The brick type permeable filter material mainly relies on the pores between the granular materials and the pores inside the material to intercept the suspended impurities, and also retains a part of COD, TN and TP while retaining suspended impurities.

\section{Results and discussion}

We conducted a new environmentally friendly gully to study the control of rainwater quality at the beginning of urban runoff. Taking SS, CODCr, TN and TP as the water quality indicators. The control of new environmentallyfriendly gullies on water quality pollutants was studied from two aspects: the same filter materials and materials with the same materials and different proportions, the same proportion and the different filter material thickness. The initial rainwater used in the experiment came from the rainwater collected from the rainy road of Ziyun Road in Hefei City. The initial rainwater quality: SS: $221 \mathrm{mg}$; CODcr: 380 mg/L; TN: 9.9 mg/L; TP: $3.1 \mathrm{mg} / \mathrm{LL}$.

\subsection{Study on the removal effect of pollutants with the same composition and different propor- tions of materials}

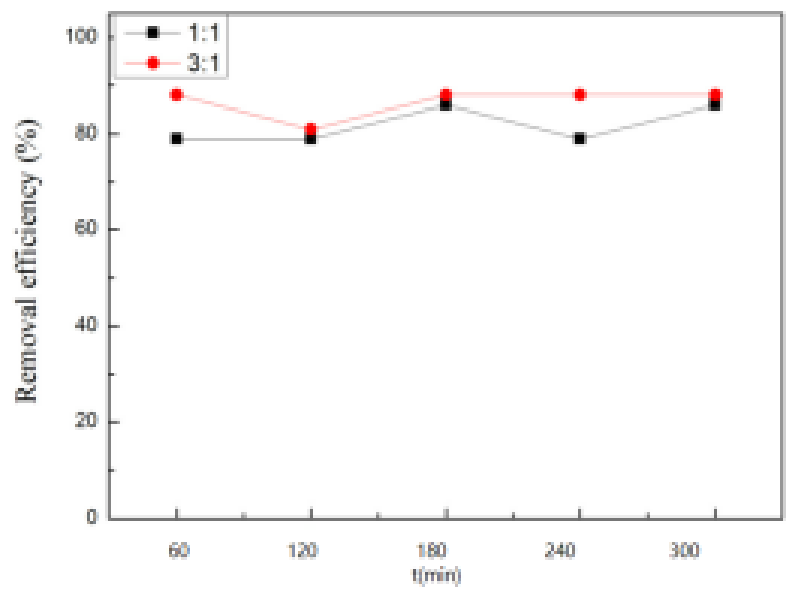

Fig. 3. (a) Effect of different proportions of materials on SS removal

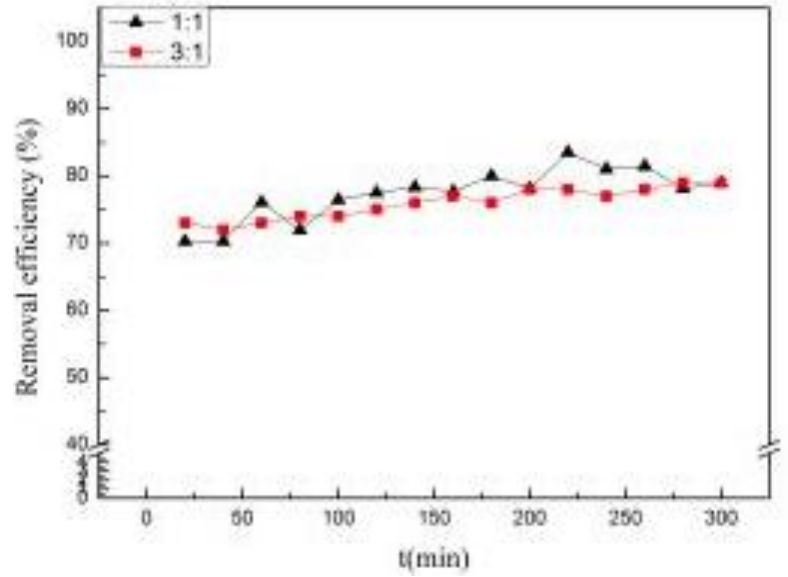

Fig. 3. (b) Effect of different proportions of materials on TN removal

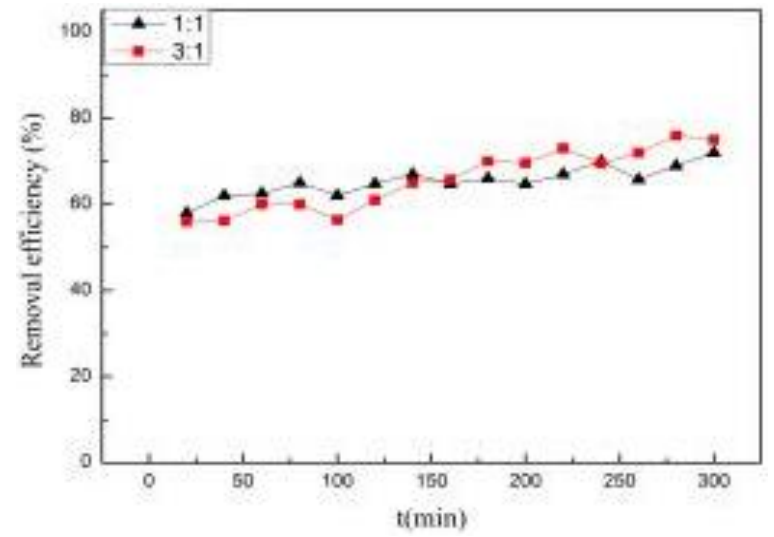

Fig. 3. (c) Effect of different proportions of materials on TP removal

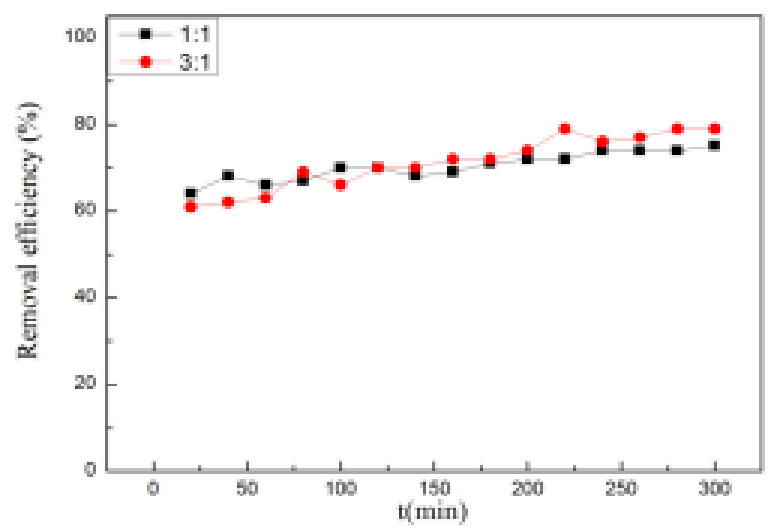

Fig. 3. (d) Effect of different proportions of materials on COD

Figure 3 (a) shows that the material ratio of 1:1 is very fluctuating at $3 \mathrm{~h}$, and then tends to rise after $4 \mathrm{~h}$, the overall removal rate is about $79 \% \sim 86 \%$; the material ratio of $3: 1$ is in time. The SS removal rate fluctuated greatly between $1 \sim 3 \mathrm{~h}$, firstly from the removal rate of $88 \%$ to $79 \%$, then from $79 \%$ to $88 \%$, and after $3 \mathrm{~h}$ the removal rate began to stabilize, which may be the beginning of the larger amount of water. After SS is transmitted, the water volume tends to be stable, and the SS removal rate also tends to be stable. The overall removal rate is about $81 \%$ to $88 \%$. The 
overall SS removal rate of the filter material with a material ratio of 3:1 over time is higher than the filter material with a material ratio of $1: 1$. The comparison of the two materials in the statistical pairwise data ratio mean value shows that the two materials are different.

Figure 3 (b) shows that the total nitrogen removal rate of the filter material with a material ratio of $1: 1$ fluctuates greatly. When the time is from $230 \mathrm{~min}$ to $300 \mathrm{~min}$, the contaminant may block the material due to the passage of time during the removal process, resulting in a slight removal rate. Decrease, the overall removal range is about $70 \% \sim 83 \%$; while the total nitrogen removal rate of the filter material with a material ratio of 3:1 has a small fluctuation, but the overall trend shows a steady upward trend, and the removal range is about $72 \% \sim 79 \%$. In the comparison of the statistical means pairwise data ratio mean value, the ratio of filter material to material ratio of $1: 1$ material ratio to material ratio of 3:1 filter material has reached a significant level of difference in the initial rainwater removal efficiency.

Figure 3 (c) shows that the total phosphorus removal rate of the filter material with a material ratio of $1: 1$ is fluctuating but generally shows an upward trend, the overall removal range is from $59 \%$ to $73 \%$; and the total material ratio of the material ratio is $3: 1$. Phosphorus removal rate fluctuated slightly, but the overall trend was also rising. The removal range was about $57 \% \sim 77 \%$. Compared with the material ratio of $1: 1$, the filter material fluctuated greatly. The material ratio of 1:1 was before $180 \mathrm{~min}$. The overall removal rate is higher than the material ratio of $3: 1$, but the overall removal rate of the material with a material ratio of $1: 1$ after 180 minutes is significantly lower than the material ratio of $3: 1$. The comparison of the mean ratios of the two pairs of statistical data in the two materials indicates that there is no difference between the two materials.

Figure 3 (d) shows that the COD removal rate fluctuates greatly when the filter material with a material ratio of $1: 1$ is in the $0 \sim 150 \mathrm{~min}$ removal rate, while the COD removal rate fluctuates less after the time of $150 \mathrm{~min}$, but the overall removal rate increases. Trends, it is obvious that the removal rate ranges from $64 \%$ to $75 \%$; the filter material with a material ratio of $3: 1$ fluctuates greatly, but the overall trend is still rising, and the removal rate ranges from $61 \%$ to $79 \%$. The filter material removal rate range of 1:1 material ratio is larger and the fluctuation is larger. After the time of $125 \mathrm{~min}$, the overall COD removal rate of the material ratio of $3: 1$ is obviously higher than that of the material ratio of $1: 1$. The comparison of the mean ratios of the two pairs of statistical data in the two materials indicates that there is no difference between the two materials.

\section{Conclusion}

In view of the current gully conditions, research has been carried out on the design of the gully shell structure and the production of filter bag materials. The new environmentally-friendly gully has the functions not currently available in the existing gully: (1) it can reduce the SS and COD problems of rainwater pollutants; (2) it has anti-mosquito and deodorization functions; (3) it is innovative in terms of the structure of the outer casing.

In summary, under the condition of different filter material ratio and filter bag thickness, the new environmental protection rainwater outlet has better removal effect on various pollutants in the initial rainwater, and the removal rate of SS reaches over $80 \%$ and the rate of the total nitrogen removal is above $75 \%$, and the removal rate of COD and total phosphorus is over $70 \%$. The new gully has a good effect on the removal of pollutants from rainwater, which reduces the pollution of the initial rainwater to a certain extent. It provides a new choice for the construction of rainwater outlets in urban construction and is of great significance in the construction of sponge cities.

\section{Acknowledgement}

This work was supported by the Science and Technology Major projects of Anhui Province (18030801106,16030801118 and 18030801104), the Natural Science Major Research Projects of Anhui Education Department (KJ2016SD14, KJ2017ZD40 and KJ2017ZD41), the National Natural Science Foundation of China (61873003), the Scientific Research Start-up Foundation for Introduction of Talent, Anhui Jianzhu University (2016QD113).

\section{References}

1. W. Che, J. Q. Li, M. Beijing: China Building Industry Press, (2006).

2. T. Y. Huang. J. Value Eng. 29, 1 (2010).

3. B. Li, S. F. Zhang, L. Hu. J. China Municl Eng, 5, 3 (2012).

4. Y. Wu. J. Chinese Construc. 6, 2 (2012).

5. X. N. Li. D. 75 (2015).

6. F. Wang, Q. Gao, X. H. Lu, Y. L. Lu, P. E. Leng. J. Chinese J. Vec. Bio. Con. 29, 4 (2018).

7. R. T. Bannerman, D. W. Owens, R. B. Dodds. J. Water Sci. Techno. 28, 9 (1993).

8. J. Liu, W. Y. Dong, Y. L. Li. J. Construc. Econo. 2, 4 (2014).

9. Y. Chang, J. Shanxi Archi. 30, 2 (2004).

10. X. F. Li. J. R Traf. Eng. 28, 3 (2010).

11. Z. H. Yuan. D 70 (2017). 